\title{
HILOS, CABLES Y CONECTORES: LOS HUMILDES COMPONENTES EN LA ELECTRÓNICA
}

\section{WIRES, CABLES AND CONNECTORS: THE HUMBLE COMPONENTS IN ELECTRONICS.}

\section{AUTORES \\ Arturo Pérez París: Alumno de la Escuela Politécnica de la Universidad de Alcalá arturo.perez@aol.com}

\section{CURRÍCULUM VITAE}

Alumno de la Escuela Politécnica de la Universidad de Alcalá de Henares (España). Ingeniero de Soporte Técnico en Kone Elevadores

\section{RESUMEN}

Siempre he creído que son los detalles más insignificantes los que determinan que las cosas funcionen o no. Por otro lado, es de todos conocido el hecho de que la principal causa de los defectos y averías en nuestras máquinas es el deterioro, y por esto el mal conexionado de los sistemas está en el origen de muchos fallos. Con todo ello, un punto fundamental a la hora de implementar cualquier equipo es las consideraciones que hagamos sobre los cableados a realizar y los conectores a emplear. Esta es la razón que me ha llevado a escribir el siguiente artículo, donde expongo, de manera muy superficial, qué materiales aislantes recubren los cables, qué es un conductor y qué es un cable, cómo influye la densidad de corriente en la sección a emplear y que tipo de conductores nos encontraremos en el mercado. Por último se tratará el tema de los conectores. 


\title{
PALABRAS CLAVE
}

Hilos - Cables - Conectores - Electrónica

\begin{abstract}
I always thought that are the smallest details that determine things work or not. On the other hand, is well known that the main cause of defects and damage to our machines is the deterioration, and therefore bad wiring systems is at the root of many failures. All in all, a fundamental point when any team is implementing the considerations to make about the wiring and connectors to make use. This is the reason that led me to write the next article where I discuss, on a superficial level, what insulation covering the wires, which is a driver and what is a cable, how it influences the current density used in the section and what kind of drivers we will be in the market. Finally, we address the issue of the connectors.
\end{abstract}

\section{KEY WORDS}

Threads - Cables - Connectors - Electronics

Siempre he creído que son los detalles más insignificantes los que determinan que las cosas funcionen o no. Por otro lado, es de todos conocido el hecho de que la principal causa de los defectos y averías en nuestras máquinas es el deterioro, y por esto el mal conexionado de los sistemas está en el origen de muchos fallos. Con todo ello, un 
punto fundamental a la hora de implementar cualquier equipo es las consideraciones que hagamos sobre los cableados a realizar y los conectores a emplear. Esta es la razón que me ha llevado a escribir el siguiente artículo, donde expongo, de manera muy superficial, qué materiales aislantes recubren los cables, qué es un conductor y qué es un cable, cómo influye la densidad de corriente en la sección a emplear y que tipo de conductores nos encontraremos en el mercado. Por último se tratará el tema de los conectores.

Comencemos por los materiales aislantes que recubrirán nuestros cableados. Sabemos que, por su constitución, no todos los cuerpos permiten de igual forma el paso de la corriente eléctrica. La experiencia nos enseña que hay ciertos materiales por los que la corriente eléctrica circula con gran facilidad; otros, en cambio, necesitan de ciertas condiciones para favorecer el paso de los electrones, y aún existe otro grupo, que recibe el nombre de aislantes, compuesto por materiales que se oponen casi totalmente al paso de la corriente eléctrica. Se podrían incluir en este grupo la madera, el plástico, el papel, la porcelana, los barnices aislantes, etc. Se ha dicho casi totalmente, porque, aun sin favorecer el paso de los electrones, en ciertas condiciones especiales no existe material aislante, por ejemplo, con elevada humedad, para grandes tensiones, etc. No obstante, se consideran no conductores, es decir, aislantes en condiciones generales.

Las propiedades dignas de mención en los aislantes son muy diversas, según se examinen sus aspectos eléctricos, químicos o físicos.

Como propiedades eléctricas tenemos:

Resistividad: Es la medida de la resistencia eléctrica de un material en general, dentro de unas características de ambiente y tamaño determinadas. Suele medirse en $\mathrm{W} \mathrm{m}$, o en $\mathrm{W} \mathrm{cm}$. 


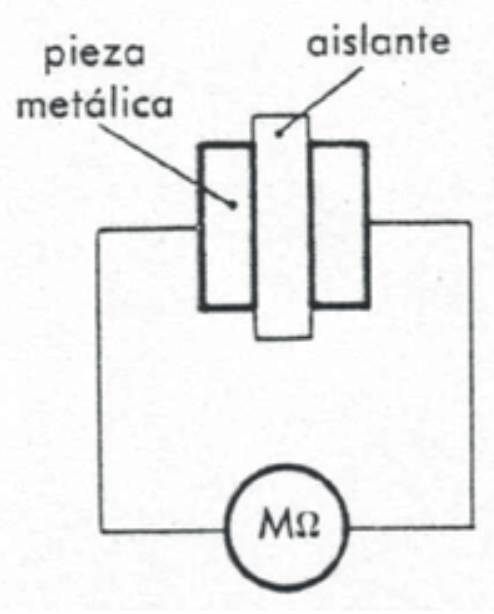

Rigidez dieléctrica: Es la oposición que presenta un aislante a la ruptura eléctrica, o lo que es equivalente, la intensidad del campo eléctrico homogéneo bajo el cual se produce la conducción. Para realizar el ensayo de rigidez de un material, se sitúa éste entre dos placas, llamadas electrodos, y el conjunto se conecta a una fuente de alimentación variable, como aparece a continuación:

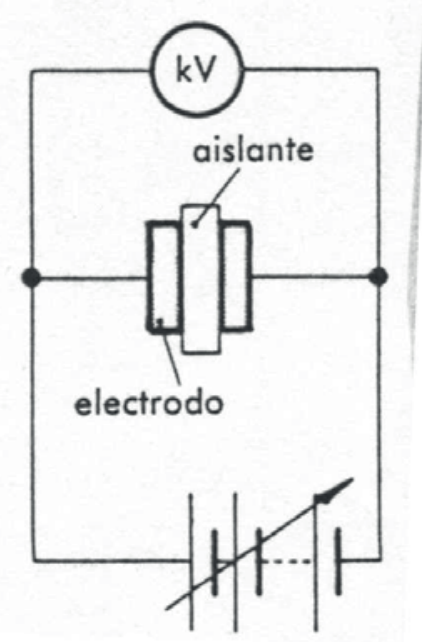


La intensidad del campo a que se somete el cuerpo aislante por ensayar, cuando la configuración de electrodos es plano-paralela, vale:

Siendo $\mathrm{V}$ la tensión existente entre las placas y d la distancia entre los electrodos. Se irá aumentando la tensión $\mathrm{V}$ de la fuente de alimentación y se llegará a un valor en el que se producirá la ruptura del aislante. La tensión a la que se ha producido este fenómeno recibe el nombre de tensión de ruptura o perforación. La intensidad de campo de perforación valdrá:

Como hemos mencionado anteriormente, la intensidad de campo de ruptura recibe el nombre de rigidez dieléctrica y se mide en $\mathrm{KV} / \mathrm{mm}$; cada material posee su valor de rigidez.

Coeficiente de seguridad: Los aislantes no trabajan con valores próximos a las tensiones de ruptura. Siempre se les da un margen para evitar el peligro que supondría la perforación.

Las normas regulan estos valores y obligan a los fabricantes a dar este coeficiente, que varía según las condiciones de trabajo a que vaya a estar sometido el aislante, con el fin de asegurar el correcto funcionamiento de las instalaciones y la debida protección a los usuarios de las mismas.

El coeficiente de seguridad viene dado por la relación existente entre la tensión de perforación y la tensión de trabajo: 
y suele oscilar entre 2,5 y 7 .

Con frecuencia los aislantes se emplean como soporte. En tales casos han de ser resistentes a la tracción, a la comprensión, al choque, etc. Como quiera que, a veces, estos mismos aislantes han de ser moldeados y trabajados mecánicamente, conviene conocer sus características antes de tales operaciones.

Los aislantes han de ser inalterables en las condiciones ambientales de trabajo. Hay que conocer su comportamiento frente a ácidos, álcalis, y disolventes.

También ha de conocerse su resistencia a la oxidación, pues, si ésta se da con frecuencia, se deteriora solamente la capa superficial a su contacto con el oxigeno atmosférico y pierde sus características aislantes. A través de las características térmicas, se mide la temperatura máxima de utilización de un aislante. Hay un código que reglamenta la gama de temperaturas.

Aislante clase $\mathrm{O}$, hasta $90^{\circ} \mathrm{C}$.

Aislante clase A, hasta $105^{\circ} \mathrm{C}$.

Aislante clase E, hasta $120^{\circ} \mathrm{C}$.

Aislante clase B, hasta $130{ }^{\circ} \mathrm{C}$.

Aislante clase $\mathrm{F}$, hasta $155^{\circ} \mathrm{C}$.

Aislante clase $\mathrm{H}$, hasta $180{ }^{\circ} \mathrm{C}$.

Aislante clase $\mathrm{C}$, por encima de $180^{\circ} \mathrm{C}$.

Las instalaciones eléctricas se realizan mediante materiales que permiten el paso de la corriente eléctrica sin ofrecer dificultad. Son, pues, imprescindibles para el desarrollo de la tecnología eléctrica y electrónica, ya que sin ellos sería imposible cualquier realización. Como es obvio, la estructura molecular de los materiales 
difiere notablemente de unos a otros, por ello no todos los cuerpos permiten el paso de la corriente eléctrica con la misma facilidad.

A los que menor oposición presentan al paso de la corriente se les denomina conductores. De ellos, destacan el oro y la plata; pero su elevado precio hace que no se empleen como tales, salvo en aparatos de precisión. Las aleaciones de plata se usan para contactos en aparatos de maniobra.

Los materiales empleados comúnmente son el cobre y el aluminio, peores conductores, pero mucho más baratos. Estos conductores se presentan de diversas maneras: en forma de hilos, de varillas, de cables, de pletinas, etc. Se llaman hilos cuando el conductor es cilíndrico y no pasa de $4 \mathrm{~mm}$ de diámetro; se emplean en las instalaciones interiores. Se denominan varillas cuando el conductor sobrepasa $4 \mathrm{~mm}$ de diámetro; se utilizan en líneas de distribución. Son cables cuando el conductor se compone de varios hilos de menor sección enrollados en espiral; se emplean en instalaciones interiores y en líneas eléctricas de mucha intensidad. Se llaman pletinas cuando el conductor es de sección rectangular; se emplean en bobinados y cuadros de maniobra y distribución. Las diferentes secciónes de los conductores está justificada, si se piensa que cada instalación precisa de valores de corriente eléctrica distintos.

Al ser recorridos por la corriente eléctrica, los conductores, aumentan su temperatura. Además, cuanto mayor es el valor de la intensidad que circula por un conductor, mayor es el calentamiento, y antes puede llegar su deterioro o la avería del circuito. Sería fácil comprender este razonamiento con un símil hidráulico, suponiendo que el conductor es una tubería y la corriente eléctrica el agua que por ella circula. Si el caudal de agua aumenta hasta valores superiores a los que la tubería puede transportar, ésta termina por romperse. 
De lo expuesto nace un nuevo concepto, el de densidad de corriente eléctrica, que es el cociente que resulta de dividir el valor de intensidad de corriente eléctrica que recorre un conductor por la sección geométrica del mismo. Se expresa por la fórmula

Suelen fijarse, para cada tipo de instalación, los valores máximos de corriente eléctrica que pueden circular. La densidad de corriente admisible depende de varios factores. Conviene señalar, entre ellos, estos dos:

Los hilos desnudos pierden más fácilmente el calor que los hilos aislados; por consiguiente, soportan mayor intensidad de corriente que estos últimos.

Si se duplica el radio, la sección aumenta cuatro veces; si se triplica o cuadruplica el radio, la sección crece nueve y dieciséis veces respectivamente.

Las limitaciones que hace el Reglamento Electrotécnico para Baja Tensión son las de los párrafos 30.14.1 y 30.14.2.

Se dice que los conductores están desnudos cuando no llevan ningún recubrimiento aislante; y aislados, cuando llevan dicho recubrimiento. El aislamiento de los conductores se hace con diversos materiales. Se dice que es textil cuando se recubre el conductor con una capa trenzada de algodón. Si el recubrimiento es doble, una cinta va cruzada con la otra, y se llama doble capa; esta capa puede impregnada con una sustancia aislante.

Se dice que un conductor es vulcanizado, cuando va recubierto de una capa de caucho vulcanizado. Esta capa suele ir recubierta de otra capa textil impregnada. Cuando el conductor vulcanizado es de cobre, éste debe llevar un baño de estaño que le proteja de la corrosión de la capa aislante. La fabricación de materiales plásticos 
resistentes al calor ha permitido la construcción de cables de aislante plástico; el más empleado en la actualidad es el cloruro de polivinilo (PCV).

Otros aislantes muy utilizados son: goma sintética SBR, goma butílica, Piperol, etc. Comercialmente se fabrican muchos tipos de conductores, de acuerdo con el uso al que vayan a ser destinados. He aquí algunos:

Tipo IAAR:

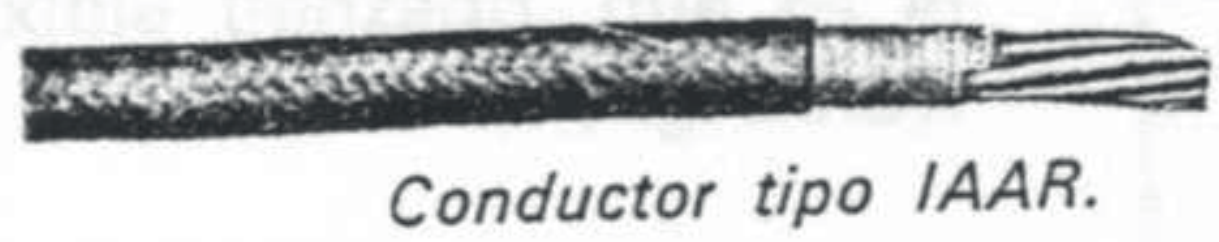

A propósito para instalaciones a la intemperie. Se presenta en dos formas: como hilo para secciones de 1 a $10 \mathrm{~mm} 2$, y como cable para las de 6,3 hasta $100 \mathrm{~m} 2$. Siempre es de cobre recubierto con dos capas textiles, la interior en espiral y la exterior trenzada. Ambas van barnizadas con barniz intemperie.

Tipo IKB:

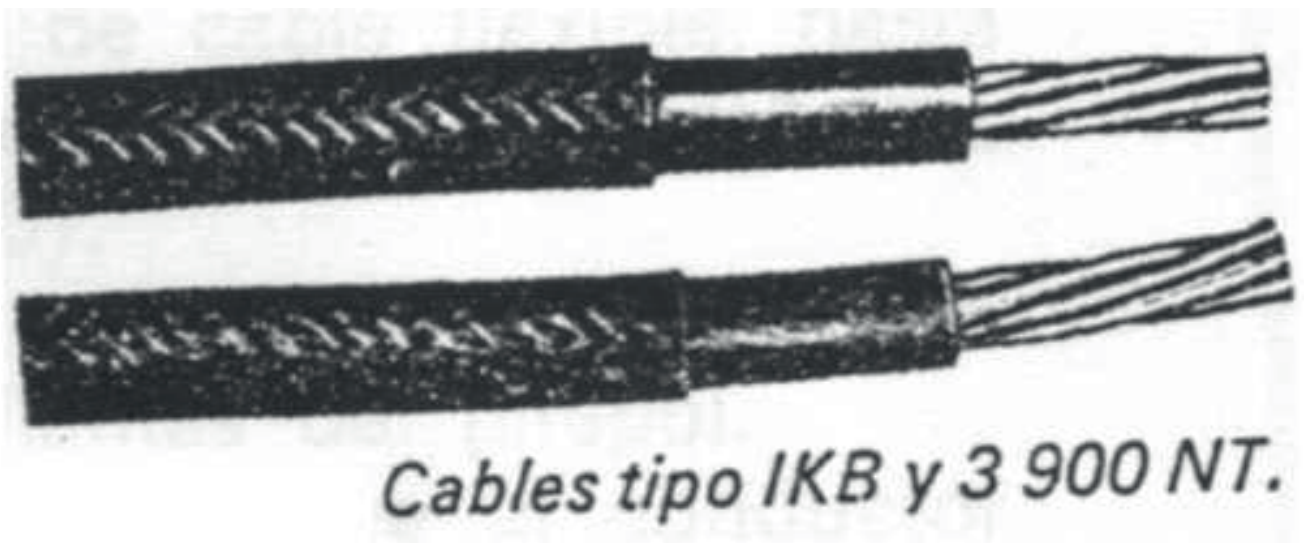


Es un conductor para instalaciones interiores, cuya sección puede ser de 1 a $10 \mathrm{~mm} 2$ para hilo y de 6,3 a $100 \mathrm{~mm} 2$ para cable. El conductor va estañado y recubierto por una capa de caucho vulcanizado sobre la que lleva una trenza textil negra.

Tipo 3900 BT:

Es una variante del tipo IKB, de mucha mayor utilización actualmente. Se compone de hilo o cable de cobre con protección metálica, tubo de goma vulcanizado y, a partir de

$16 \mathrm{~mm} 2$, cinta de papel sobre el cable y cinta de tela sobre la goma aislante. Exteriormente lleva una capa trenzada textil y barniz negro. Tanto el tipo IKB como el BT están siendo sustituidos en la actualidad por el Polipol, de mejores características.

Tipo GTR:

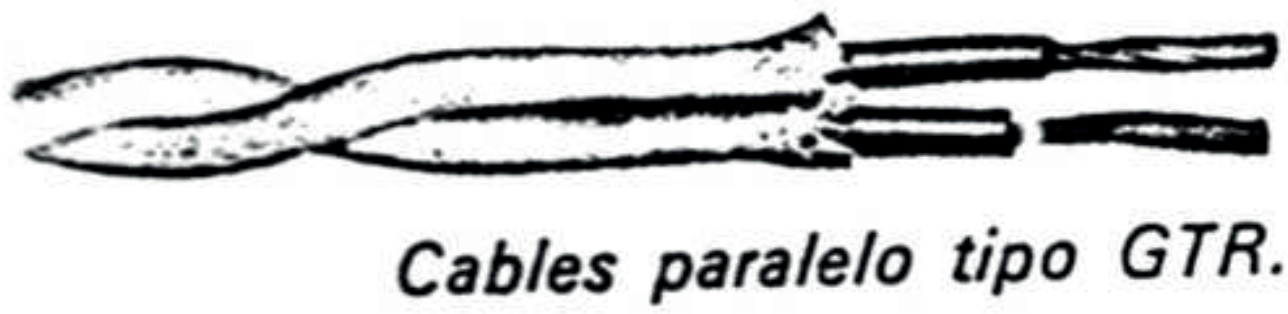

Es un conductor en espiral visible, compuesto por dos cables de cobre estañados, ambos con protección de caucho vulcanizado y capa textil blanca. Casi no se usa.

Tipo Pirepol: 


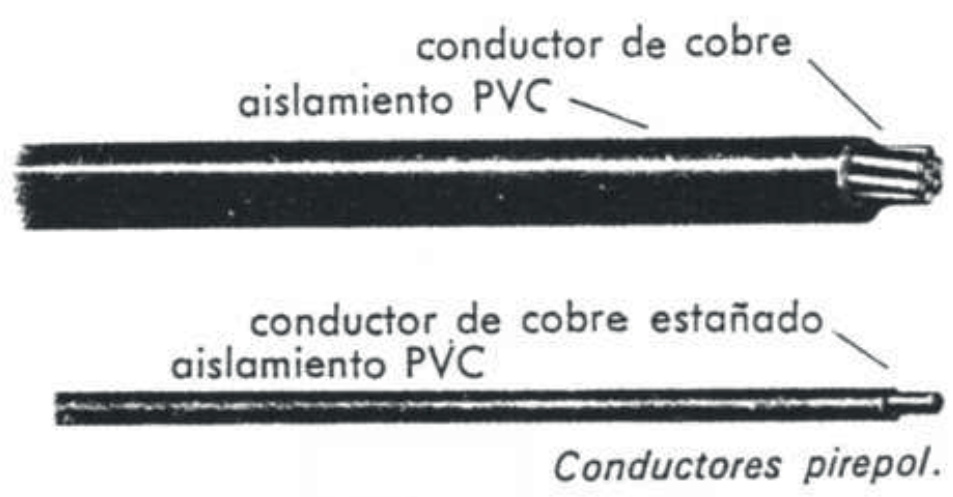

Se fabrica en forma de hilo y de cable. Al pirepol en hilo se le denomina rígido; se fabrica con un solo conductor aislado, con una capa de plástico (PVC) , y se emplea para secciones de 0,16 a 6,3 mm2. También se fabrica en pirepol rígido con secciones de 10 a $100 \mathrm{~mm} 2$, pero en forma de cable.

El pirepol se clasifica en dos grupos: flexible y extraflexible. El pirepol flexible es un cable compuesto por hilos finos de cobre, lo que le permite una gran flexibilidad. Va recubierto por una capa aislante común de cloruro de polivinilo. Puede estar formado por uno, dos o tres conductores. Es el denominado paralelo flexible. En el tipo extraflexible, los hilos que forman el cable son mucho más finos, con lo que se aumenta su flexibilidad. Sobre la capa de cloruro de polivinilo, llevan una trenza textil común.

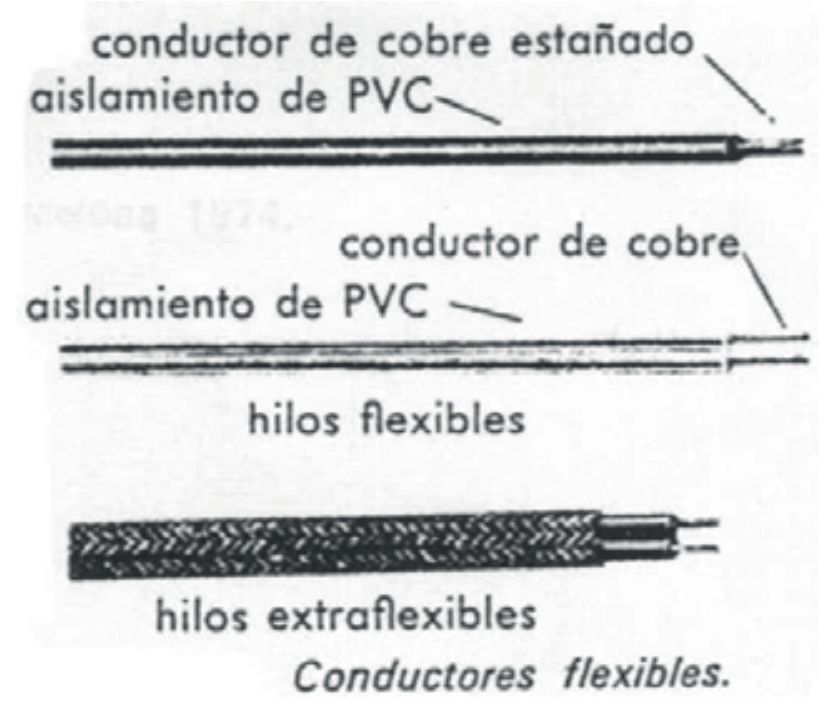


Conductores tipo pirepol, para aplicaciones especiales:

1. Pirepol-gas:

Es el tipo de cable denominado manguera. Se utiliza para acometidas a máquinas que, por cualquier circunstancia, no van bajo tubo. Se fabrican bipolares y tripolares. Consta cada conductor de un cable flexible de cobre con aislante de PVC y una cubierta exterior común, de protección, en plástico de alta calidad.

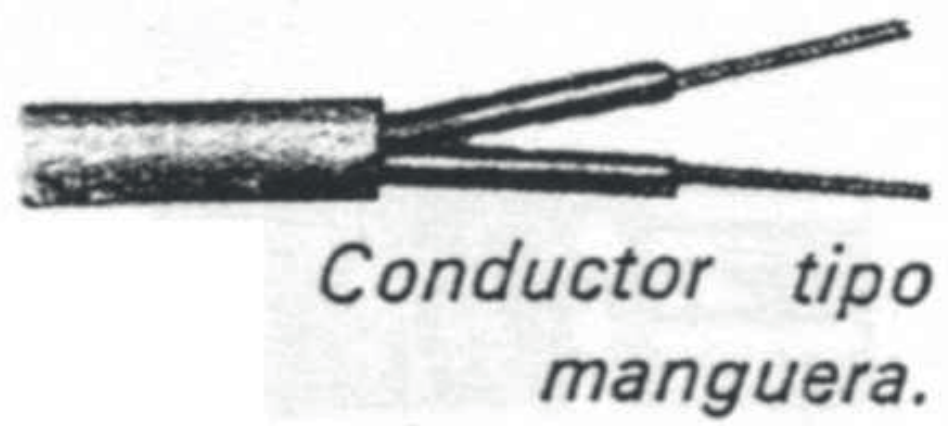

Se fabrican de $2 \times 0,63$ a $2 \times 6,3 \mathrm{~mm} 2$ para bifilares; y de 3 × 0,75 a 3 × $25 \mathrm{~mm} 2$ para trifilares.

\section{Pirepol concéntrico:}

Se utiliza para acometidas. Consta de dos conductores colocados concéntricamente. El interior es de hilo rígido de cobre y lleva una funda aislante de PVC; sobre ésta va enrollada una capa de cobre flexible trenzado, que es el segundo conductor. Todo ello está cubierto por plástico de alta calidad.

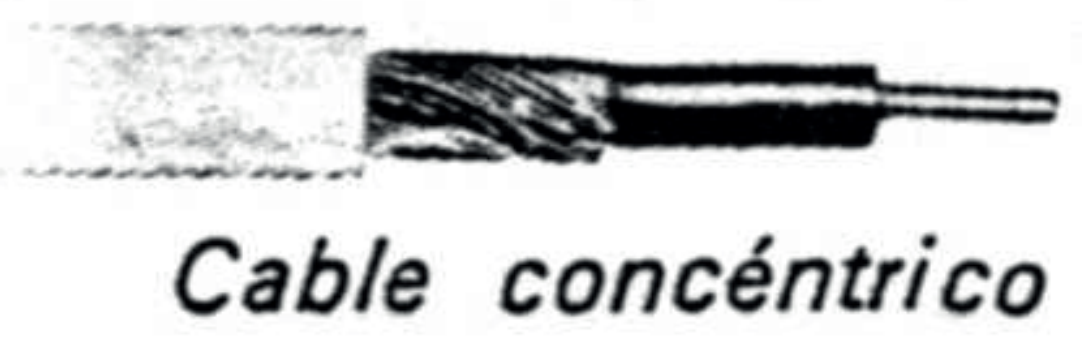




\section{Pirepol-nam:}

Es un conductor de cobre flexible, cuya cubierta aislante es muy gruesa. Se emplea en las instalaciones de encendido del automóvil.

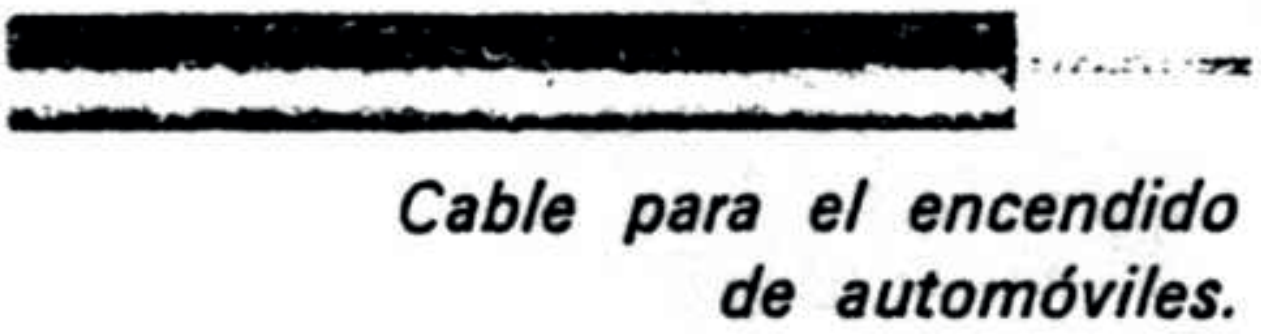

4. Pirepol-p:

Es el tipo denominado antihumedad. Se fabrica con uno, dos y tres conductores. En su construcción es similar al pirepol-gas, sólo que este tipo es de forma plana. Se construye con hilo rígido, en vez de cable flexible, hasta $4 \mathrm{~mm} 2$. A partir de 6,3 hasta $100 \mathrm{~mm} 2$ para los unifilares y hasta $25 \mathrm{~mm} 2$ para los bifilares y trifilares, en forma de cable.
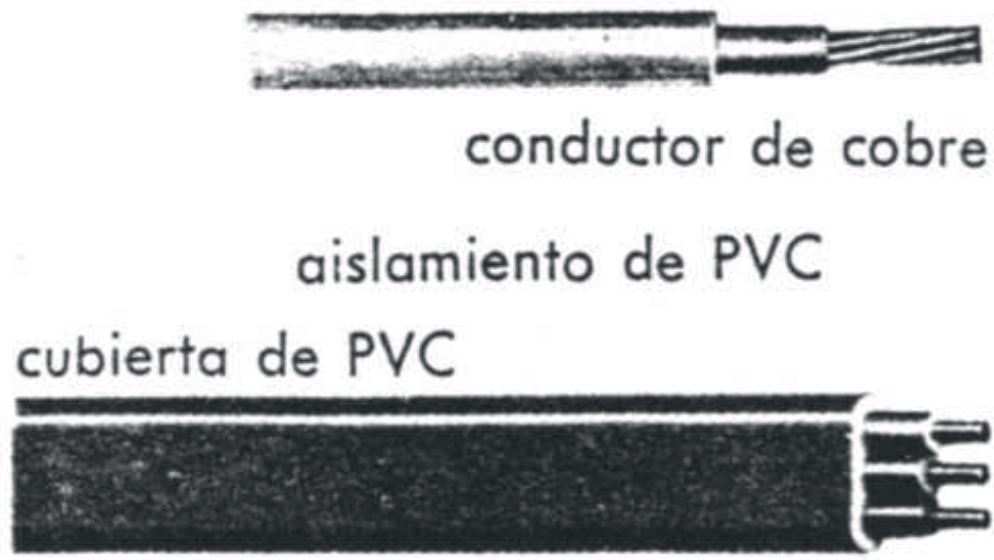

Cable antihumedad. 
También existe el tipo llamado polipol que coincide, en todas sus características constructivas, con los tipos correspondientes del pirepol. La diferencia de este conductor radica en que lleva, entre el conductor propiamente dicho y la capa aislante, un aislante intermedio transparente de PVC. Su tensión de aislamiento es, por tanto, más elevada, siendo de $1000 \mathrm{~V}$ para el pirepol y de $4000 \mathrm{~V}$ para el polipol.

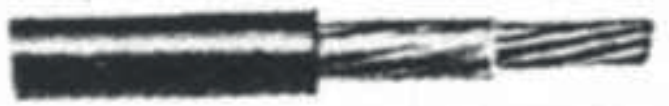

\section{polipol normal}

\section{polipol concéntrico}

\section{Conductores tipo polipol.}

Cuando se desean conectar conductores a aparatos eléctricos, o conductores entre sí, normalmente la conexión se hace a través de elementos auxiliares que facilitan esta tarea. Estos elementos son:

\section{Terminales}

Bornes

Regletas

Conectores

Bases y zócalos

Los cuatro primeros garantizan el contacto entre los conductores y aparatos eléctricos que unen, mientras que los dos últimos además de esta función permiten la rápida sustitución de los aparatos eléctricos a los que están conectados. 
Los terminales son elementos que se añaden al final de un cable de conexión, con el fin de unirlo a otro cable o a una regleta por medio de la presión. Esta presión es ejercida por un tornillo-tuerca:

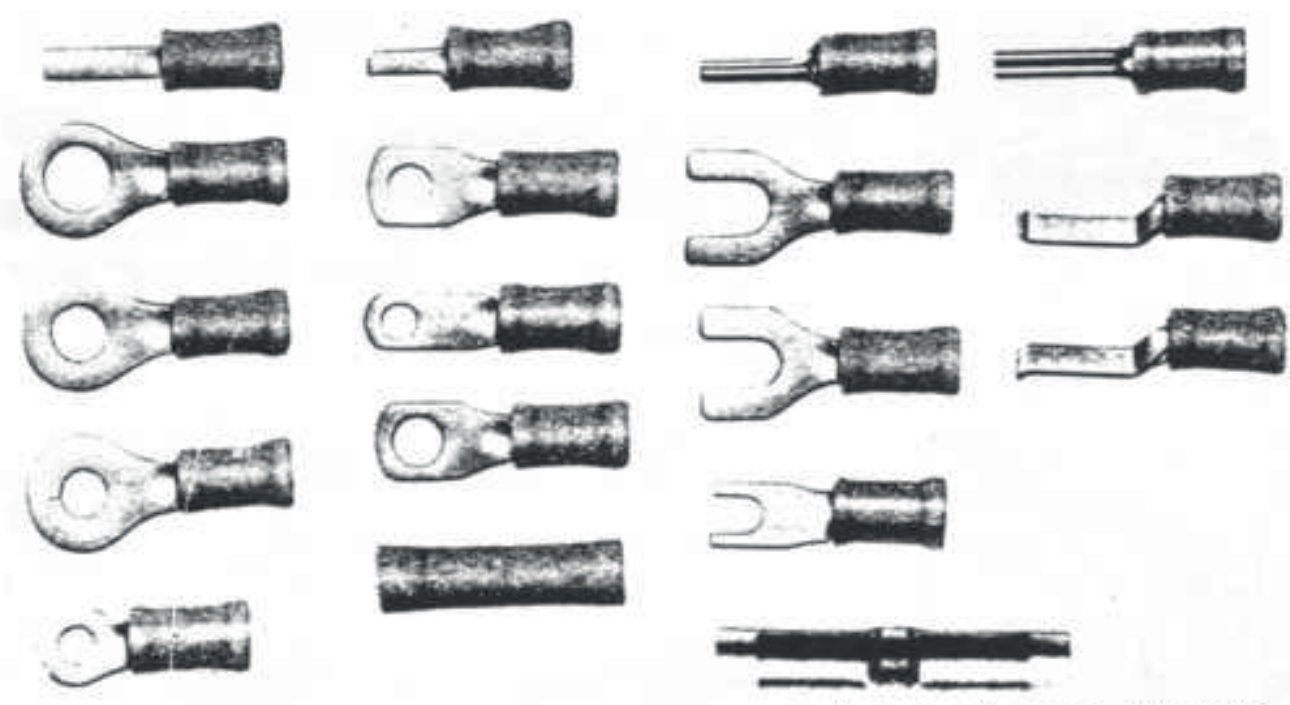

Distintos terminales, para ser conectados por medio de tornillo-tuerca. (Hay variedades de estos terminales $\sin$ aislantes.)

por la elasticidad de una presa metálica :

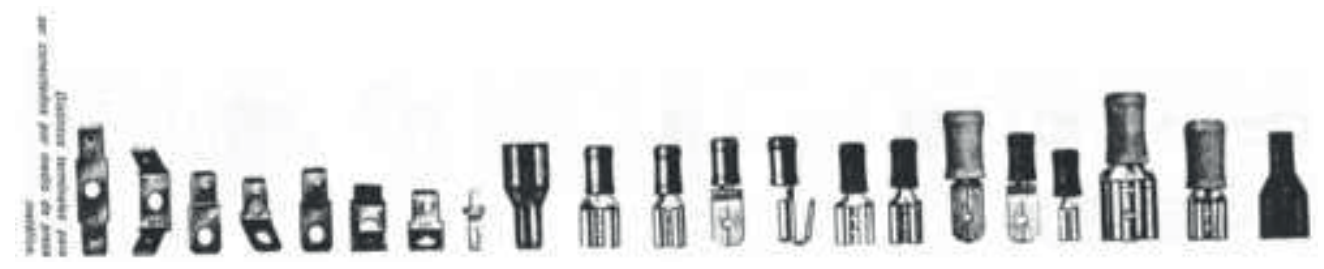

El modo de unir el terminal al conductor también puede ser de diversas formas, por soldado: 


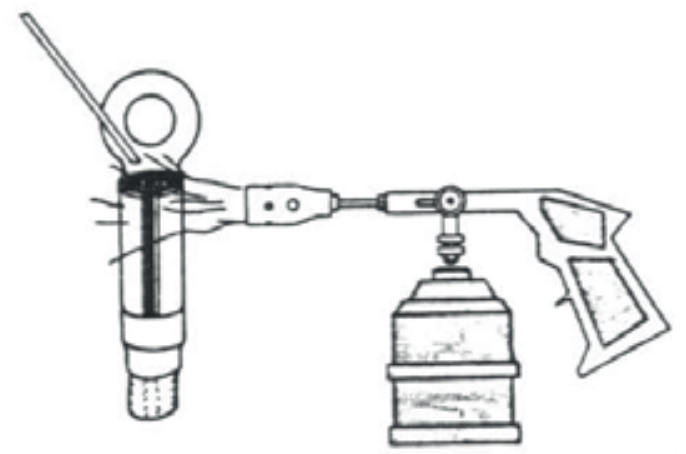

Soldadura de un terminal

a un conductor.

con tornillo de presión:

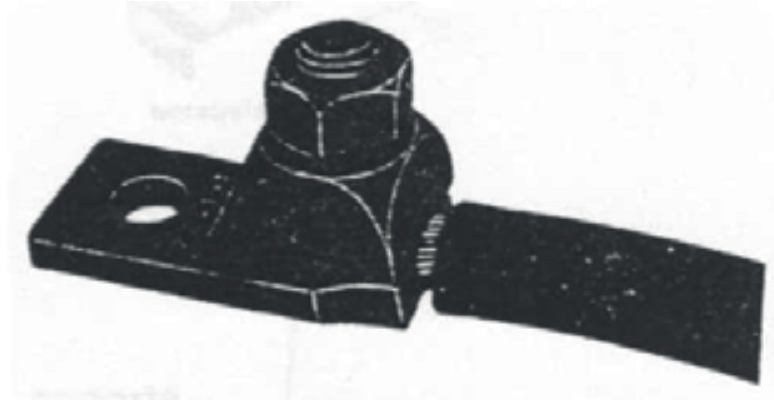

Terminal unido a un conductor por medio de tornillo de presión.

engastado:

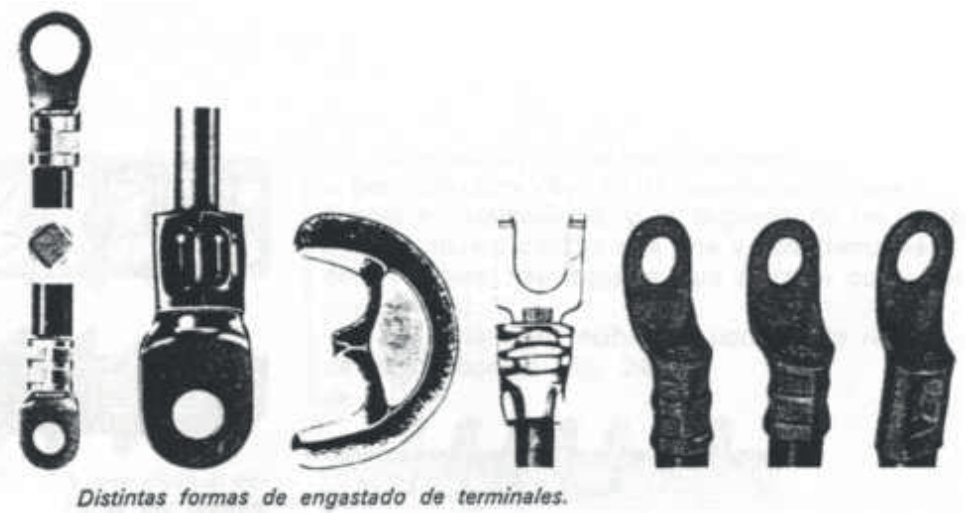


Este último método es el más usado en la unión de terminales a conductores, aunque tiene el inconveniente de precisar utillaje especial:
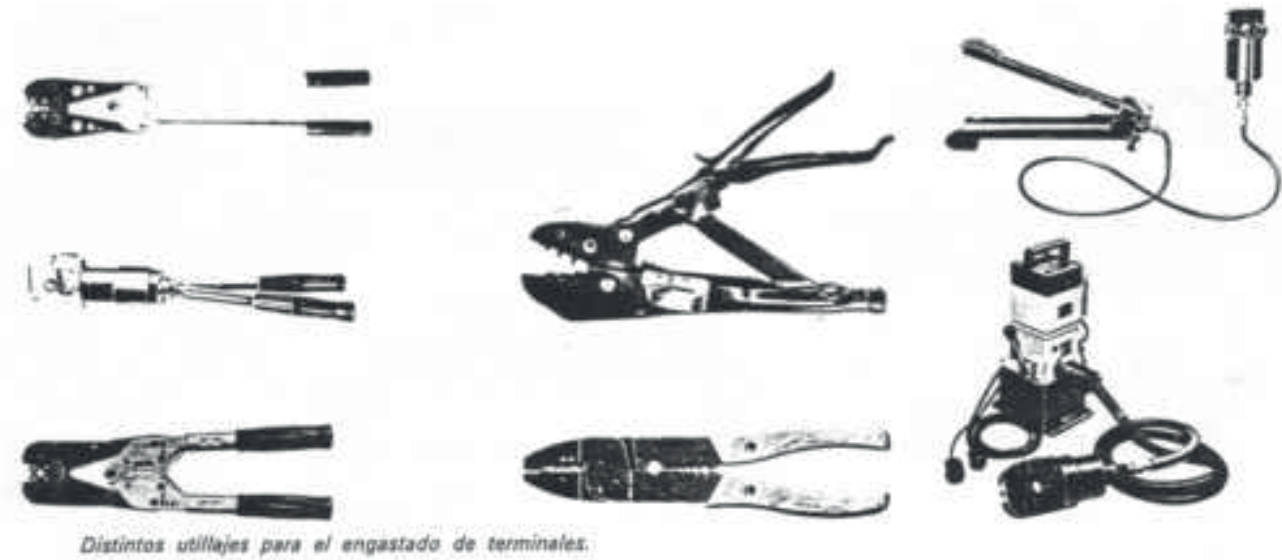

Distintos veillajes pora ol engastado de terminaies.

Los bornes son elementos destinados a la fijación de conductores, con terminal o sin él, a elementos o aparatos eléctricos. Hay gran variedad de bornes, según los usos a que son destinados:

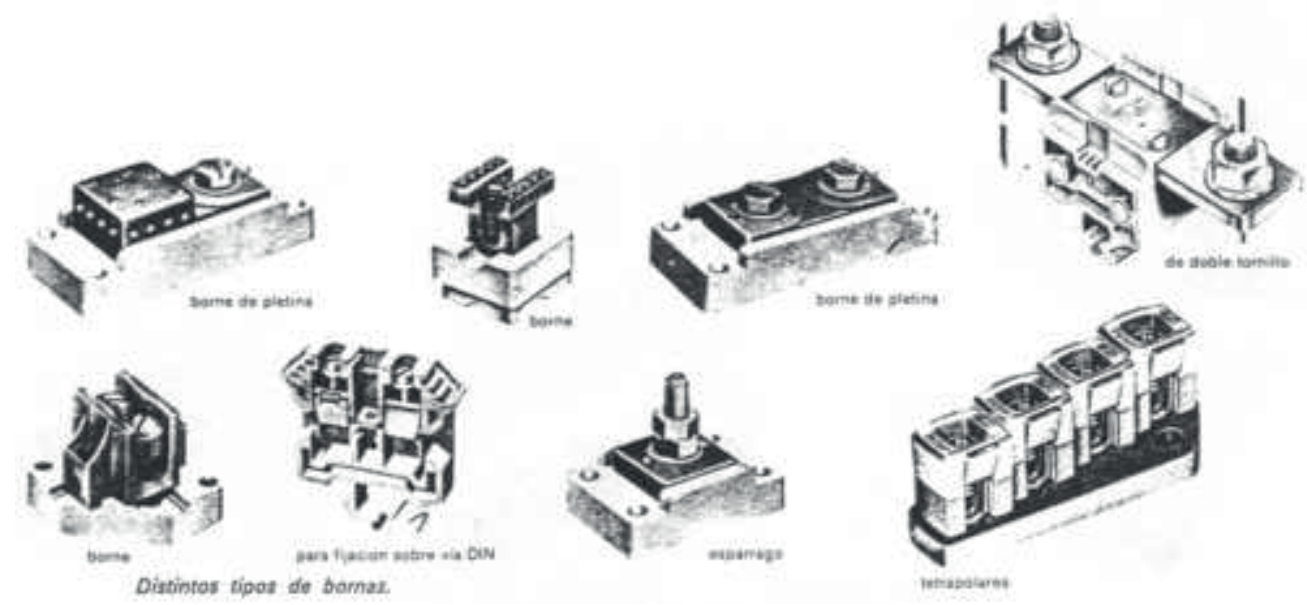

Las regletas para unir cables son unos conjuntos formados por un soporte base aislante y unos elementos metálicos de apoyo, en los cuales se realizan las diversas conexiones. La sujeción del cable a la regleta puede hacerse por presión: 

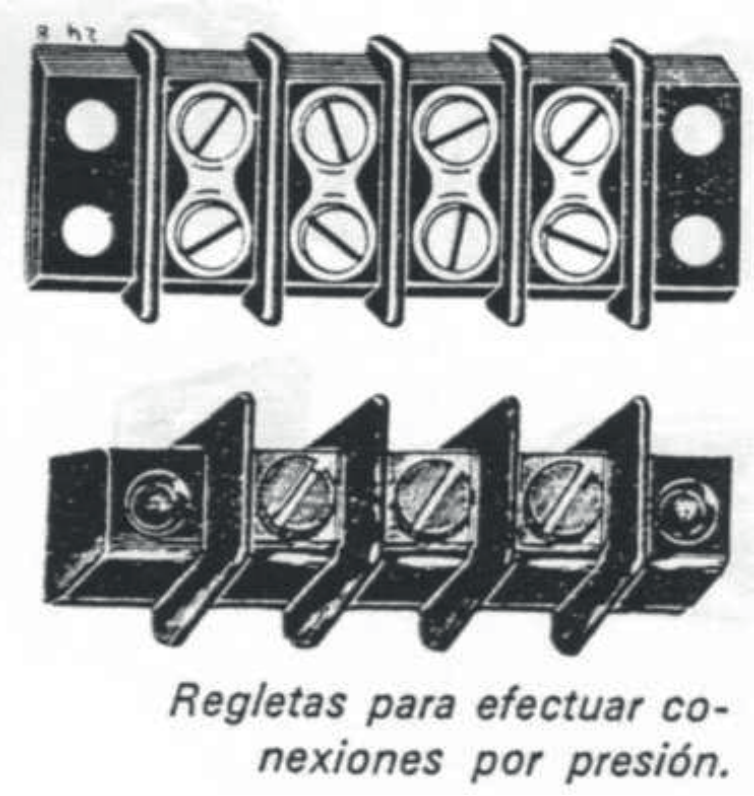

o por soldadura:
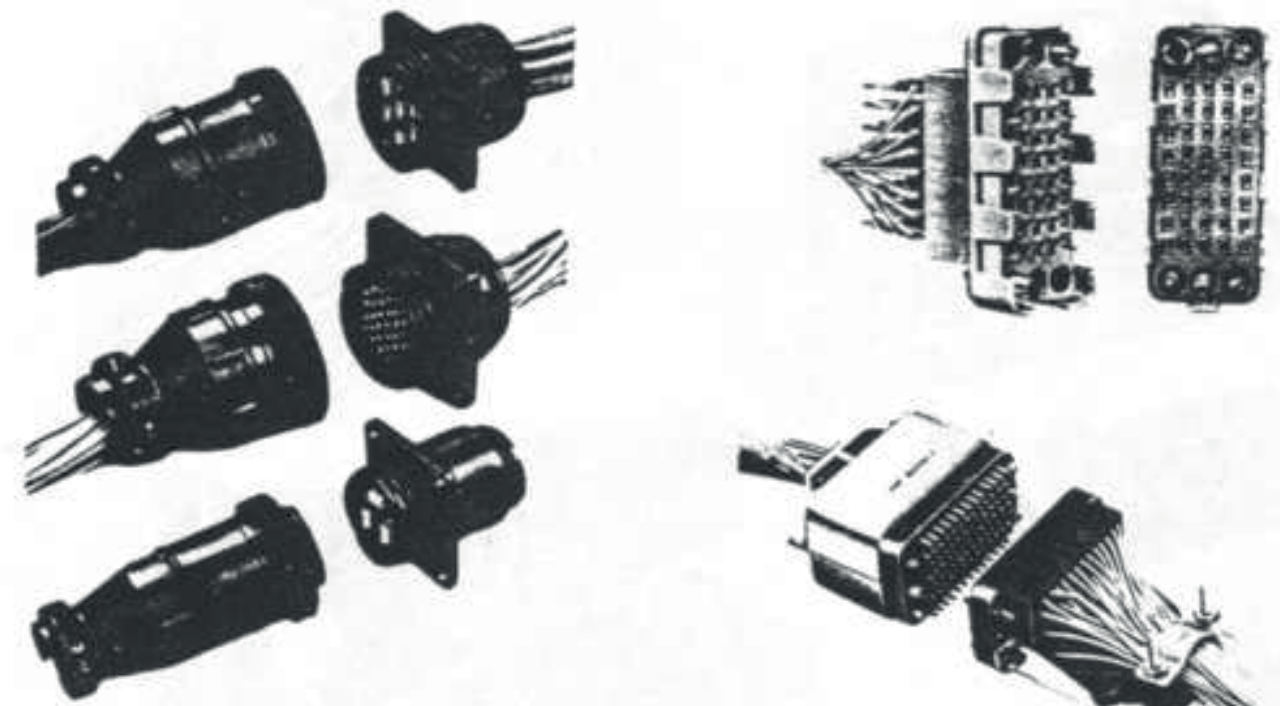

Regletas para efectuar conexiones soldadas.

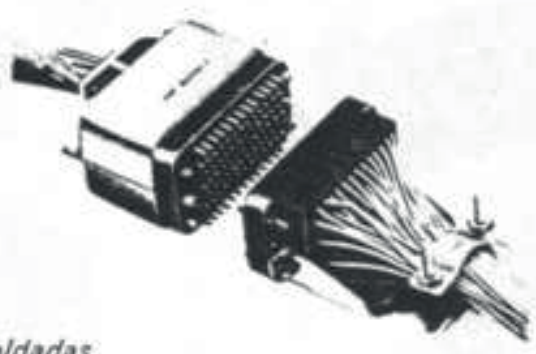

siendo la primera forma propia de las regletas utilizadas en electricidad y la segunda de las utilizadas en electrónica, sin que por ello quiera decirse que por una y otra forma sean privativas de cada especialidad. También hay regletas para efectuar contactos por presión y soldados: 

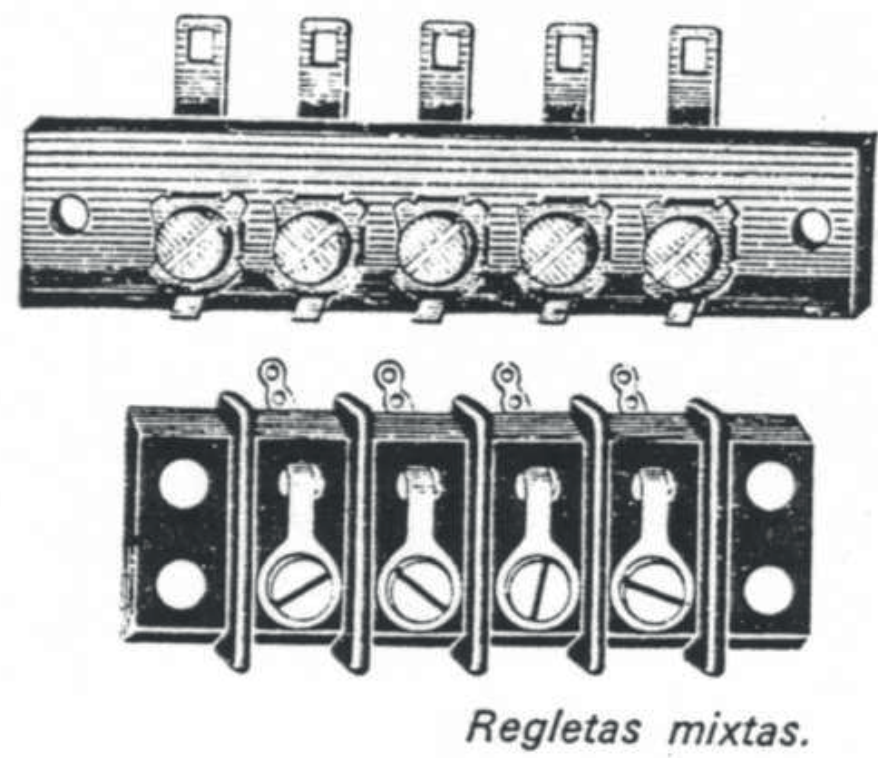

Por extensión reciben el nombre de regleta de conexiones la asociación de varios bornes:

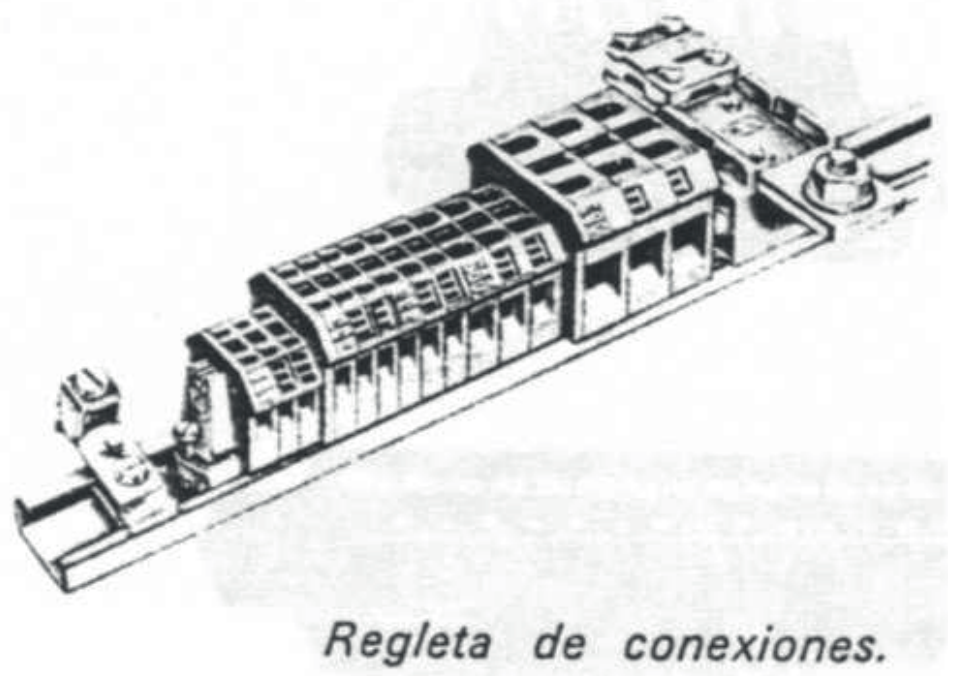

En general, se entiende por conectores aquellos elementos que facilitan la unión de dos o más conductores simultáneamente: 


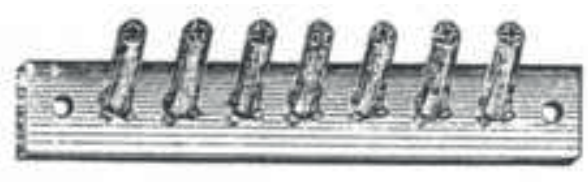

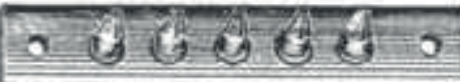

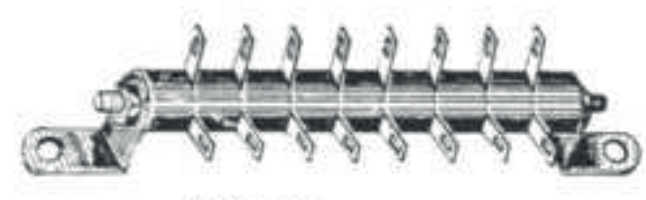

Conectores.
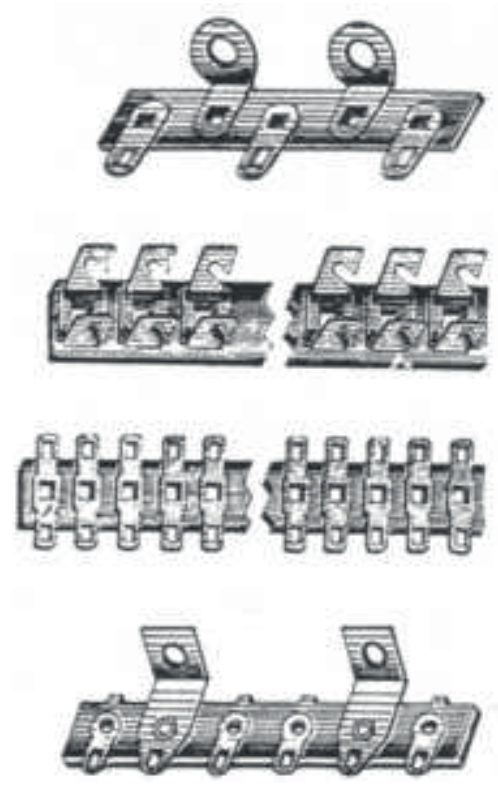

Además de la gran variedad de conectores fabricados para la industria eléctrica, hay otros que se dedican casi exclusivamente a la electrónica, dadas las especiales características constructivas:

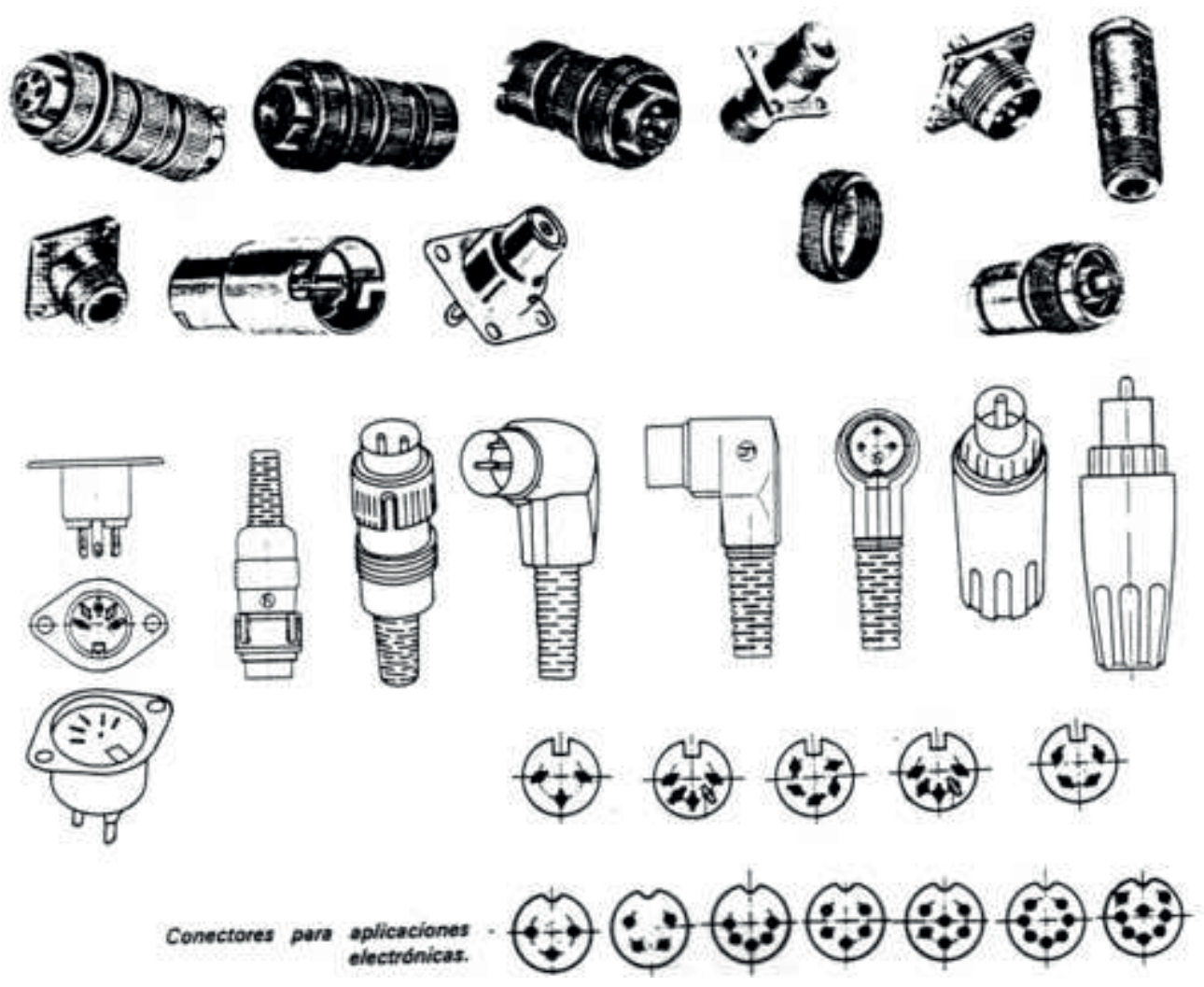


Mención especial requieren los conectores dedicados a los circuitos impresos. Estos conectores facilitan enormemente el conexionado y manipulación de las tarjetas de circuitos impresos.

Base es la parte de un componente eléctrico o electrónico, en la que se hallan los conductores, bornes o terminales que permitirán conectarlo al resto del circuito, bien por medio de conexiones directas o por medio de zócalos. Uno de los componentes electrónicos más típicos que posee base es la válvula, siendo el número de patillas de esta variable:
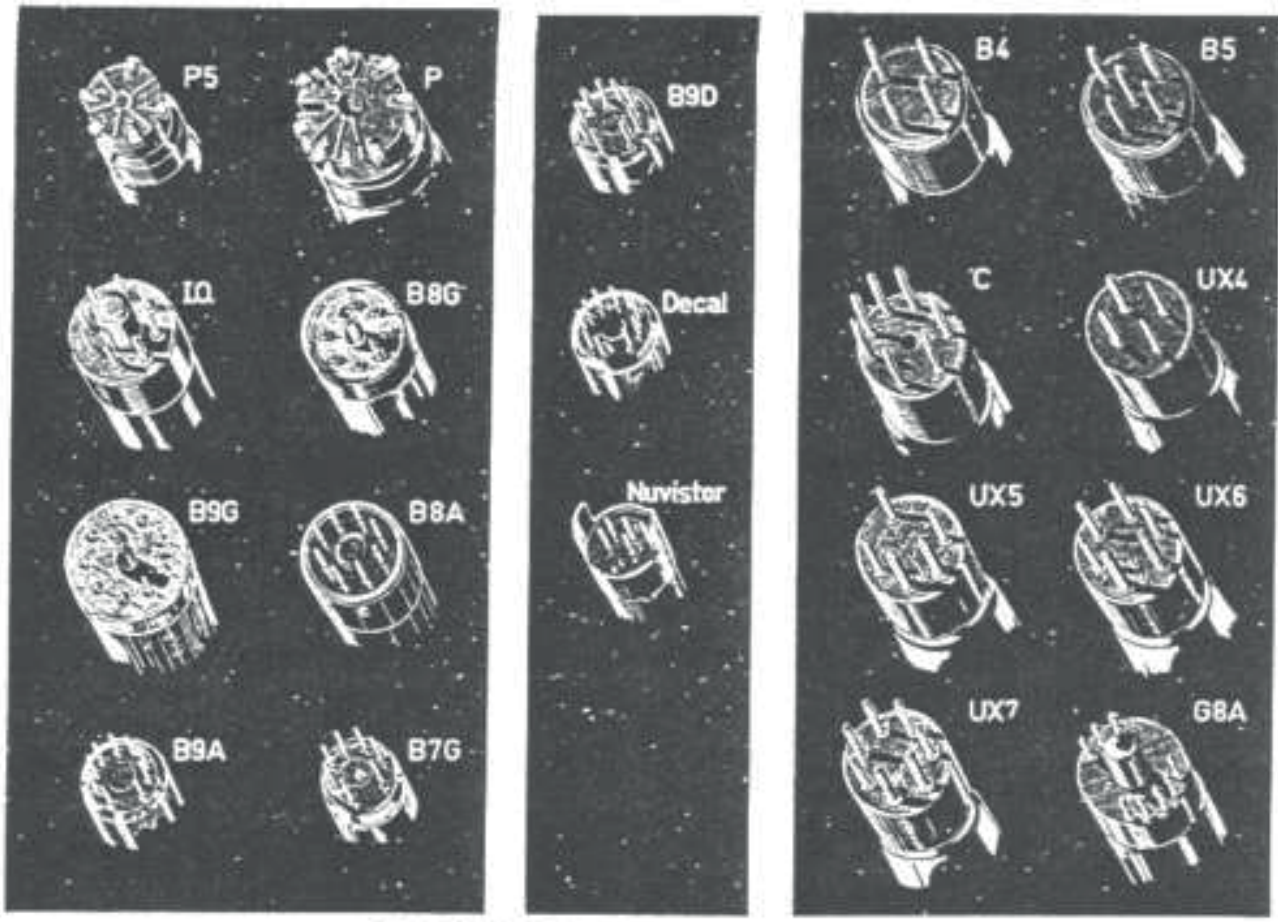

Tipos de basets ay vaivulan

Los zócalos son elementos cuya función es conectar eléctricamente y sostener mecánicamente componentes eléctricos o electrónicos, para que, de esta manera, sean fácilmente reemplazables. A continuación, pueden apreciarse distintos tipos de zócalos y usos a que son destinados. 

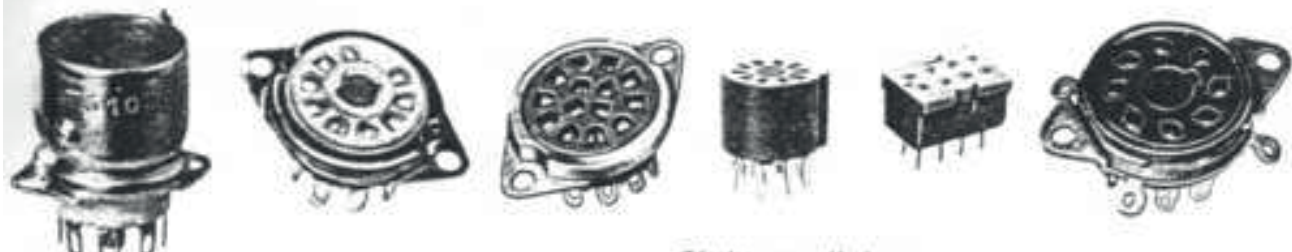

Zdealor pera velvelas.
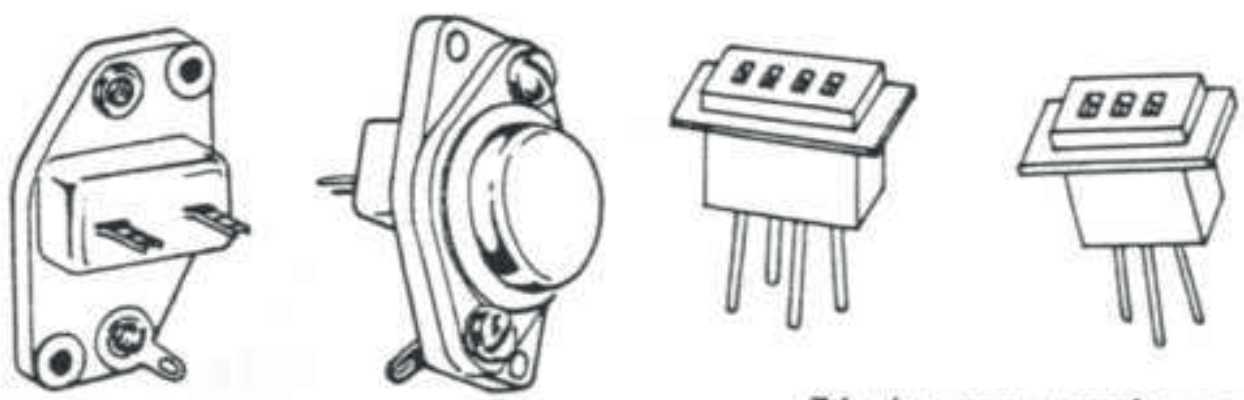

Zócalos para transistores.
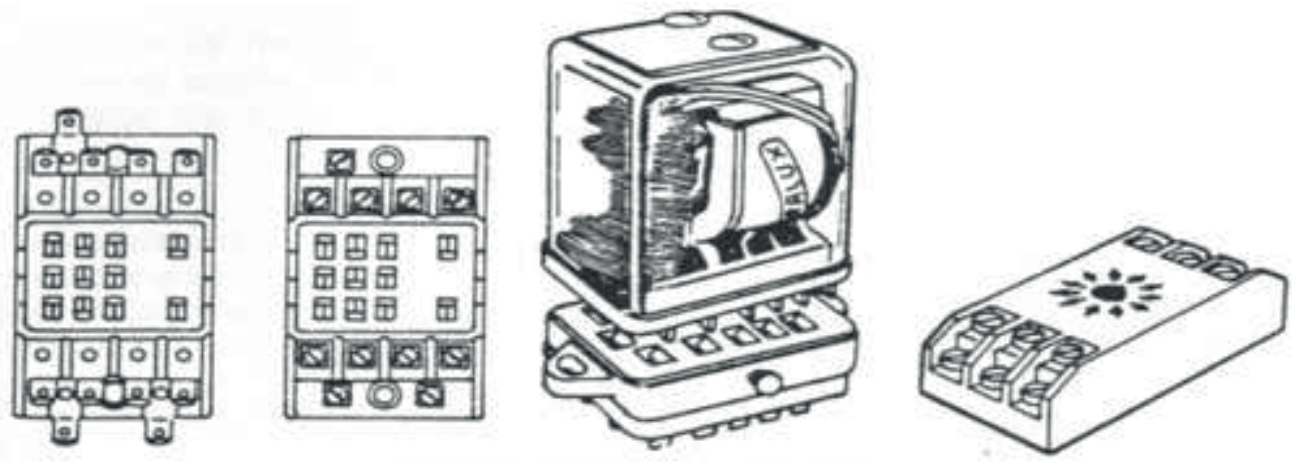

Zócalos para relés.

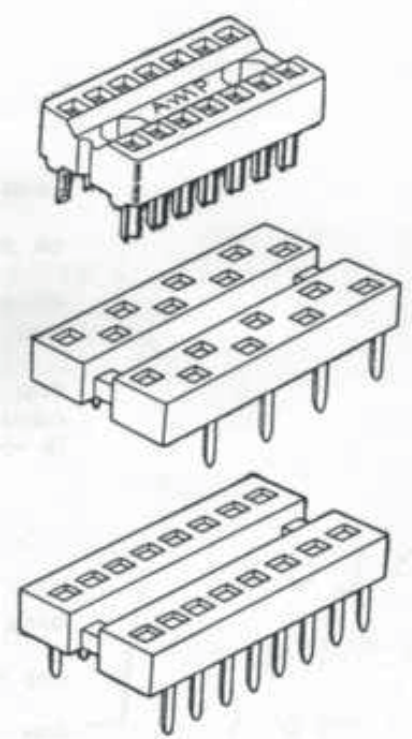

Zócalos para circuitos integrados. 
Aquí termina la presente entrega. Les espero en la próxima. Si alguien pudiera aportar sus conocimientos, para expandir éste "articulito"con nueva información, o con el fin de rebatir errores que yo haya cometido en esta exposición, o manifestar críticas (a ser posible constructivas por favor) o cualquier tipo de comentario, les animo a que se dirijan a esta magna revista, para que me "abronquen" convenientemente por "manta" (hago aquí la solemne promesa de que, en cuanto lo sepa, publicaré, si ello fuere menester, claro, aquello que se me enviare). 